\title{
Performance of chosen multiaxial cycle counting method under non-proportional multiaxial variable loading.
}

\author{
Matus Margetin ${ }^{1, *}$, and Dominik Biro ${ }^{1}$ \\ ${ }^{1}$ Slovak University of Technology, Faculty of Mechanical Engineering, Namestie Slobody 17, 81231 Bratislava, Slovakia
}

\begin{abstract}
One of the most crucial tasks in fatigue life-time estimation of components loaded with multiaxial variable amplitude loading is to correctly identify loading cycles that can be used with multiaxial damage criterions. During past years, several cycle counting methods have been proposed for multiaxial loading conditions. The aim of this text is the comparison of selected multiaxial cycle counting methods, namely Wang-Brown's method, Modified Wang-Brown's method, Bannantine-Socie's method and then a critical analysis of the obtained results. For the comparison of chosen methods, a new data set, consisting of experimentally obtained results from multiaxial non-proportional variable amplitude loading tests carried on by authors, has been used. The tested specimens were made from S355J0 structural steel and the testing procedure has been carried out on the MTS Axial/Torsion servo hydraulic testing machine. Findley and McDiarmid multiaxial criterion with Palgren-Miner summation rule have been used for fatigue life-time estimation of the tested specimens.
\end{abstract}

\section{Introduction}

Fatigue life time estimation of mechanical components working under real multiaxial variable amplitude operational loadings could be considered as an most complex task in a field of fatigue life time analysis. This complexity of fatigue life time estimation is caused by the fact that the estimation process is composed from multiple independent but crucial tasks which influence final accuracy of fatigue life time estimation.

While topics such as multiaxial damage criterions or damage accumulation rules are research subjects of many scientist and large amount of works in this fields have been published in recent years, there are not so many articles aiming on problem of loading cycle identification in variable multiaxial non-proportional loading signals. And yet, the correct identification (and followed extraction) of loading cycles from variable amplitude multiaxial loading signal is necessary step finite fatigue life time estimation. Nowadays two methods (and their variations) of cycle identification and extraction are most commonly used. They are Bannantine-Socie method and Wang-Brown method.

In this paper authors critically review two most common cycle counting method. Then modelled experiment is presented where result from experimental measurement is compared to estimated fatigue life time using mentioned cycle counting method combined with two multiaxial criterion (Findley and McDiarmid) and Palgren-Miner damage accumulation rule.

\section{Cycle extraction technique}

The stress (strain) loading signal obtained by measurement or numerical calculation needs to be transform into a form which can be used on finit fatigue life time estimation. Standardized rain flow algorithm can by used for identifying and extracting cycles from loading signal when uniaxial loading conditions occur. In this case general loading signal is transform to set of harmonic cycles with given amplitudes and mean value which has same equivalent effect on component as original signal. However, this technique can not be used for multiaxil loading cases, as opposed to uniaxial loading two independent loading signal acts on loaded component simultaneously (Fig. 1).

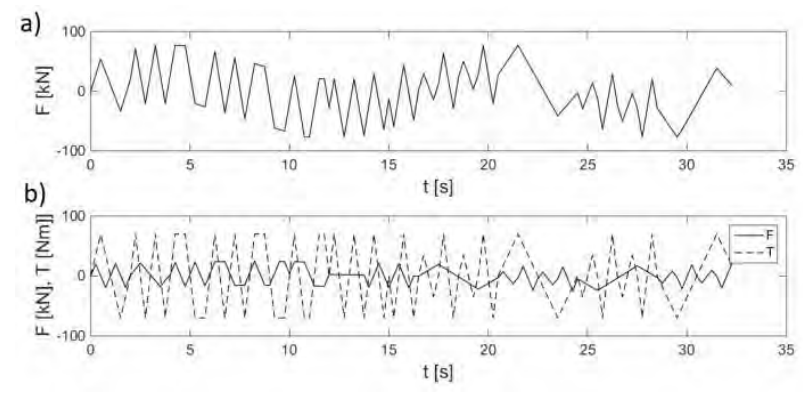

Fig. 1. a) uniaxial history; b) multiaxial history.

As a matter of fact, there is no universally used cycle counting method for fatigue life time analysis of

\footnotetext{
*Corresponding author: matus.margetin@stuba.sk
} 
component loaded with multiaxial non-proportional variable amplitude loading. Two most common used approaches to cycle identification are Bannantien-Socie method [1] and Wang-Brown method [2,3] (and theirs modifications).

\subsection{Wang-Brown method}

Wang and Brown developed the method, which can be used for any proportional or non-proportional history of strain or stress. This method identifies cycle based on an equivalent strain or stress. The von Mises equivalent stress (strain) is used to transforms multiaxial state of stress (strains) to uniaxial. The problem with using von Mises is the loss of the sign since von Mises values are always positive. For example in case $90^{\circ}$ out-of-phase loading von Mises remains constant, so it leads to the underestimating of loading condition. Because of that, the relative von Mises stress (strain) is introduced, calculated from shifted stress (strain) signals. To obtain the relative von Mises stress (strain) the loading signals have to be rearranged in the way that it starts with the points corresponding to maximal von Mises stress. The signals are then shifted by offset equal to negative value of first point (so the shifted signals starts in zero Fig. 2).

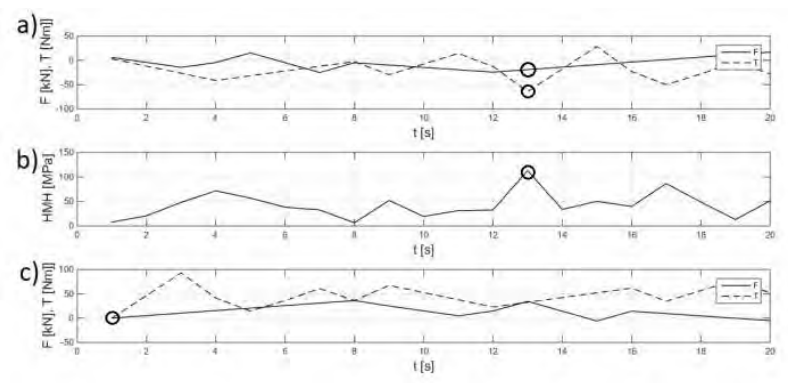

Fig. 2. a) original signal; b) von Mises stress; c) rearranged and shifted signal.

One half-cycle is generated from initial point to point with maximum value of relative von Mises strain/stress in each counting. The remained parts of the signals are then processed in the loop using the same procedure (Fig. 3)

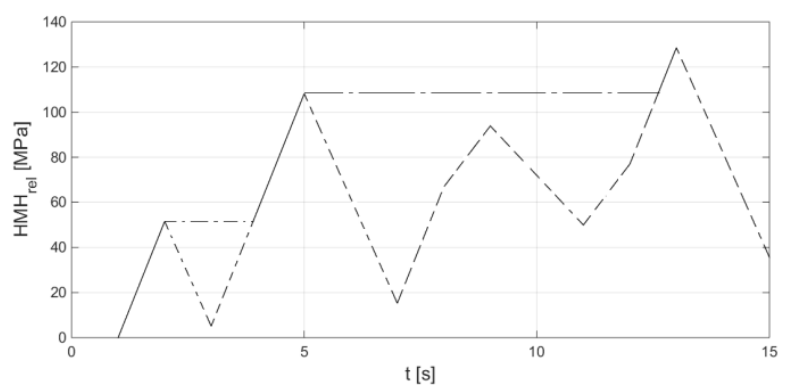

Fig. 3. Extracted half cycle - bold line; remained parts dashed line.

The main advantage of this method is that whole counting runs only once and the extracted cycles can be used with different multiaxial criterions. Even if we want to used extracted cycles with damage models based on critical plane theory, there is no needed of transformation of loading signals into the different material planes before extraction. The biggest disadvantage of this method is, that it extracts only halfcycles.

\subsection{Bannantine-Socie method}

Bannantine and Socie proposed methodology that is based on recalculating the loading signals into de plane with different orientation that it provides the normal and shear stress (strain) histories in each plane configuration. Then the modification of standard rain flow method is applied on either shear or normal stress (strain) signal, depending on chosen damage model. Te rain flow method has to keep track of events with occur in the second (non extracted) signal to capture the parameters that are needed in multiaxial fatigue criterion (Fig. 4)

Fig. 4. Tracking events on non extracted signal history.

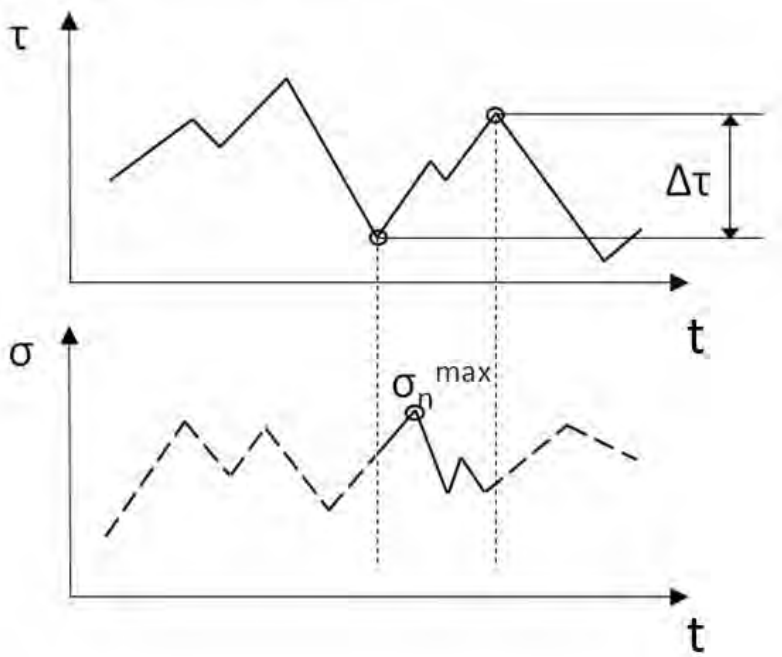

Result from this method can be directly used with critical plane damage models as the recalculation of loadings signals into the different planes is done before extraction. The main disadvantage is that this method is very time consuming as it requires run of rain flow extracting procedure in multiple planes.

\section{EXPERIMENTAL PROCEDURE}

To verify methods mentioned in previous chapter, an experiment was conducted in which experimental specimens were loaded using various multiaxial loading signals. Experiment was carried out in the Strength and Elasticity Laboratory of the Faculty of Mechanical Engineering STU. Two type of multiaxial variable amplitude loading signal have been applied on round specimens.

\subsection{The loading signals}

Two types of loading histories have been applied on testing specimens. 
The first block was composed of non-proportional combination of force and torque variable amplitude loading signals with zero mean value. The loading histories as well as loading path of the signal can be seen in Fig 5.
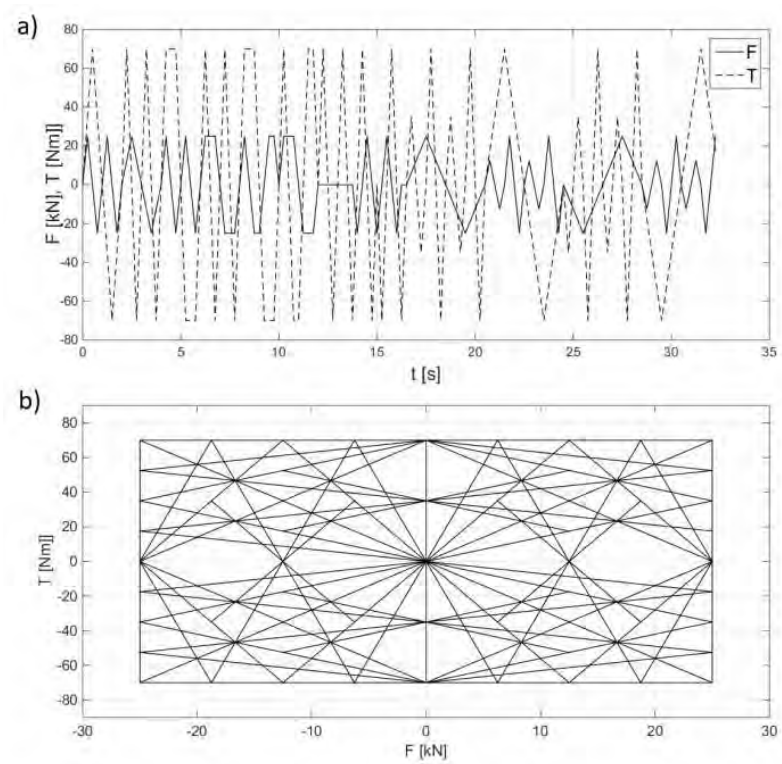

Fig. 5. a) Loading history 1 (LH1); b) Loading path.

The second block was composed from nonproportional combination of force and torque variable amplitude loading signals with variable mean value. The loading histories as well as loading path of the signal can be seen in Fig 6.
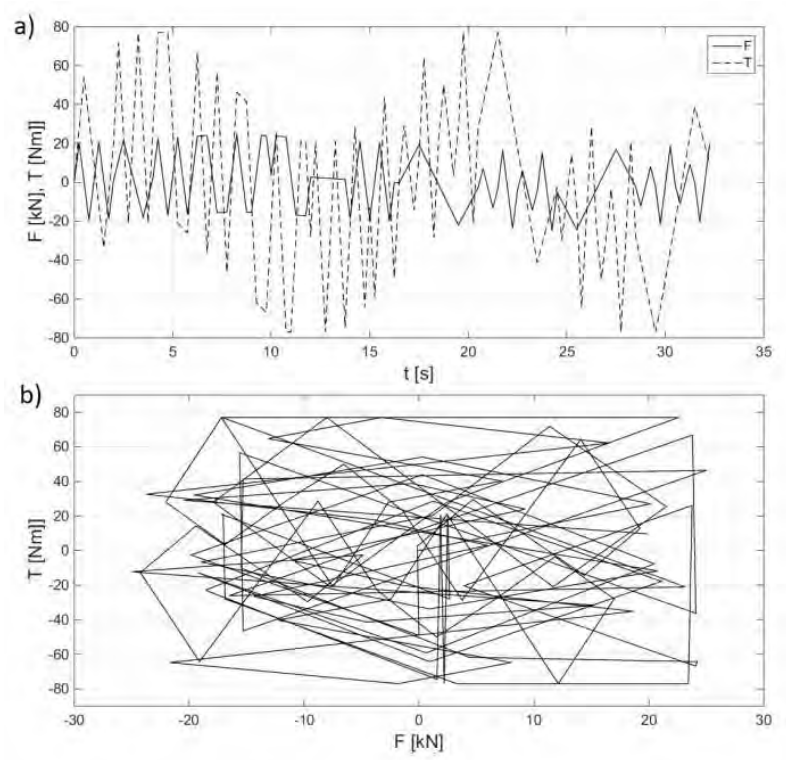

Fig. 6. a) Loading history 2 (LH2); b) Loading path.

\subsection{Testing specimens and experimental setup}

Testing specimens has been loaded with multiaxial variable amplitude loading signal on MTS Bionix 370.02 Axial/Torsional testing system. The experimental specimens were manufactured from $\mathrm{S} 355 \mathrm{~J} 2+\mathrm{C}$ structural steel. The geometry of testing specimen can be seen in Fig. 7.

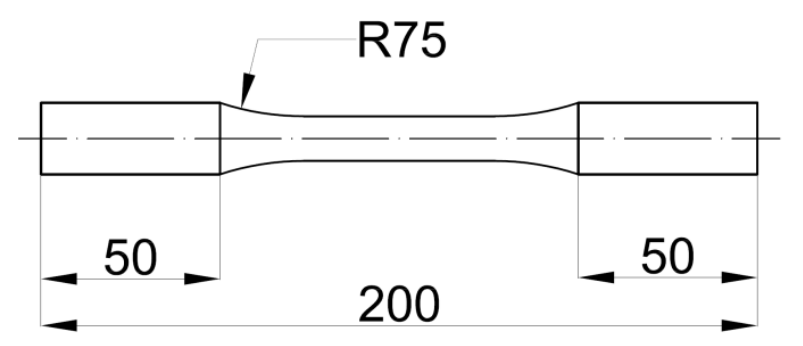

Fig. 7. Testing specimen geometry.

Chemical composition as well as static material parameters obtained from the material a-test can be seen in Table 1.

Table 1. Chemical composition and material parameters of testing specimen.

\begin{tabular}{|c|c|c|c|}
\hline \multicolumn{4}{|c|}{$\mathrm{S} 355 \mathrm{~J} 2+\mathrm{C}$} \\
\hline $\boldsymbol{\sigma}_{\mathbf{y} 02}[\mathbf{M P a}]$ & $\boldsymbol{\sigma}_{\mathbf{u}}[\mathbf{M p a}]$ & $\mathbf{C}[\%]$ & $\mathbf{P}[\%]$ \\
\hline 655 & 680 & 0,16 & 0,014 \\
\hline $\mathbf{S}[\%]$ & $\mathbf{M n}[\%]$ & $\mathbf{S i}[\%]$ & $\mathbf{C u}[\%]$ \\
\hline 0,025 & 1,31 & 0,18 & 0,12 \\
\hline $\mathbf{A l}[\%]$ & $\mathbf{M o}[\%]$ & $\mathbf{N i}[\%]$ & $\mathbf{C r}[\%]$ \\
\hline 0,018 & 0,01 & 0,06 & 0,07 \\
\hline
\end{tabular}

Te tests have been carried on in force and torque controlled mode. The number of loading block repetition prior to the initiation of the fatigue crack was determined on the basis of a continuous measurement of the deformation, and angle of the distortion, response to the loading regime of the test specimen. Failure condition of the experimental specimen was defined by the moment of the so-called "technical initiation of fatigue crack" $(0,5-1 \mathrm{~mm})$. The tests have been terminated when either maximal deformation or angle of the distortion of loading block increased by $3 \%$ in reference to previous maximums (Fig. 8).

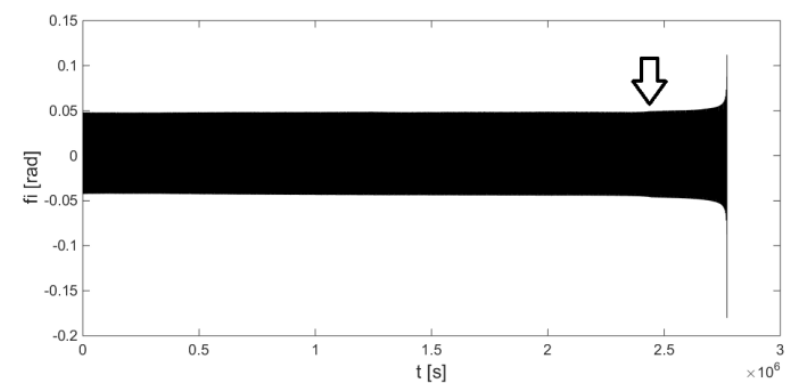

Fig. 8. "Technical" initiation of fatigue crack.

\subsection{Experimental results processing}

Fatigue life time estimations of measured specimens have been calculated from real measured specimen loading, as the real loading condition slightly differ from 
modelled loading signal histories (due to regulation imperfection).

The multiaxial criterion has to be used for final fatigue life time estimation based on extracted cycle. Two kind of multiaxial fatigue damage model have been used. Authors chose to use stress based critical plane criterion.

The first one is Findley criterion [4]. Findely propose criterion based on linear combination of shear steress amplitude and maximal normal stress acting on calculated plane. When used for finite fatigue life time estimation, the equivalent shear stress amplitude is compared to shear Basquin equation. Final form is then described by following formulation:

$\tau_{e q}=\left(\tau_{a}+k_{f i n} \sigma_{n}\right)_{\max }=\tau_{f}^{*}\left(2 N_{f}\right)^{b_{\tau}}$

The critical plane is defined as a plane with maximal damage parameter. Similarly in the case of variable amplitude loading the critical plane is the plane with maximal accumulated damage.

The second used criterion is criterion proposed by McDiarmid [5]. Similarly to Findely, McDiarmid propose criterion in form of linear combination of shear stress amplitude and maximal normal stress acting on same plane. The main difference, and reason why authors chose this criterion, is the definition of critical plane. Unlike Findley, McDiarmid propose that critical plane is plane with maximal shear stress amplitude. Then again for finite fatigue life time estimation the McDiarmid equivalent shear stress amplitude is compared to shear Basquin equation:

$\tau_{e q}=\tau_{a}+\left(\frac{k_{m c d}}{2 \sigma_{u}}\right) \sigma_{n, \max }=\tau_{f}^{\prime}\left(2 N_{f}\right)^{b_{\tau}}$

However when using this criterion for fatigue life time estimation under variable non-proportional loading. The damage in every plane has to be calculated and the final fatigue life time is determined by the plane with maximal accumulated damage.

The material parameters used in multiaxial criteria are summarized in Table 2. and are taken from [6].

Table 2. Fatigue properties.

\begin{tabular}{|c|c|c|c|c|}
\hline \multicolumn{5}{|c|}{ S355J2+C } \\
\hline $\boldsymbol{\tau}_{\mathbf{f}}[\mathbf{M P a}]$ & $\mathbf{b}_{\boldsymbol{\tau}}[-]$ & $\mathbf{k}_{\text {fin }}[-]$ & $\begin{array}{c}\boldsymbol{\tau}_{\mathbf{f}}^{*} \\
{[\mathbf{M P a}]}\end{array}$ & $\begin{array}{c}\mathbf{k}_{\text {mcd }} \\
{[\mathbf{M P a}]}\end{array}$ \\
\hline 550 & $-0,0736$ & 0,131 & 550 & 244 \\
\hline
\end{tabular}

As both criterion take into account only shear stress amplitude the methodology that take into account mean shear stress of extracted cycles has to be implemented in estimation procedure. The mean shear stress correction model proposed by Wang and Miller [7] has been used for life time estimation. The model is described by following formulation: $\tau_{a}=\tau_{a m} e^{\frac{\left|\tau_{m}\right|}{\tau_{f}}}$

Fatigue damage in each plane has been calculated using well known Palgren-Miner linear damage accumulation rule described by formula (4)

$D=\sum \frac{n_{i}}{N_{f_{i}}}$

The increment of the angle defining the evaluated plane has been set to $2^{\circ}$.

\section{Experimental results}

The methodology described in previous chapter have been used for fatigue life time estimation of testing specimens loaded with designed loading histories LH1 and LH2. Estimated results with experimental measurements are compared in following chapter. Three specimens have been tested overall. The specimen nuber 16 and specimen number $\mathrm{C} 2$ have been loaded with same loading history with different loading frequencies (loading signal has been applied two time faster on specimen $\mathrm{C} 2$ ).

\subsection{Wang-Brown method}

The comparison between measured fatigue life time and fatigue life time estimation calculated from cycles extracted using Wang-Brown method can be seen in table 4.

Table 3. Result comparison - Wang-Brown.

\begin{tabular}{|c|c|c|c|c|}
\hline \multicolumn{5}{|c|}{ Wang-Brown } \\
\hline Spec. nr. & $\begin{array}{c}\text { Load. } \\
\text { Hist. }\end{array}$ & Exp. & Findley & McDia. \\
\hline 16 & LH1 & 6520 & 11781 & 6569 \\
\hline C2 & LH1 & 6715 & 16148 & 8905 \\
\hline C4 & LH2 & 14480 & 17054 & 9584 \\
\hline
\end{tabular}

Fatigue life time estimation as a function of plane orientation for specimen n.16 (loaded with loading history 1) can be seen on Fig. 9.

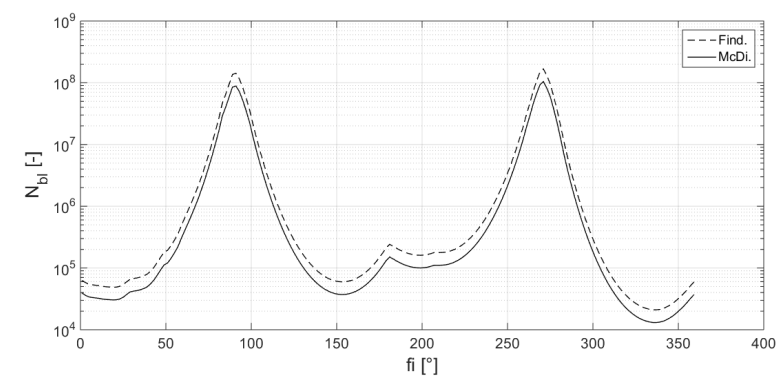

Fig.9. Fatigue life time as a function of plane orientation Wang-Brown method loading history LH1.

Fatigue life time estimation as a function of plane orientation for specimen n.C4 (loaded with loading history 2) can be seen on Fig. 10. 


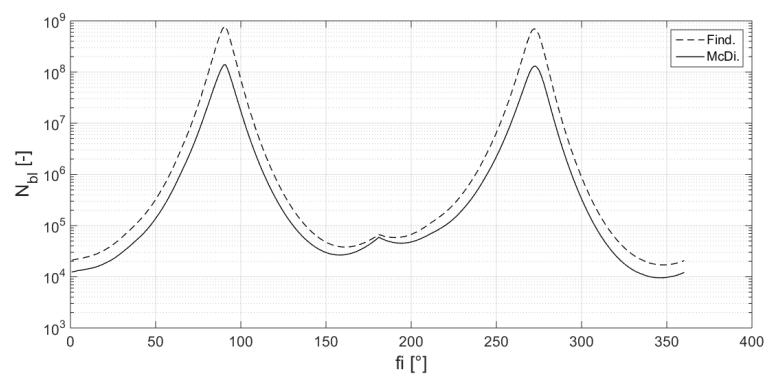

Fig.10. Fatigue life time as a function of plane orientation Wang-Brown method loading history LH2.

\subsection{Banantine-Socie method}

The comparison between measured fatigue life time and fatigue life time estimation calculated from cycles extracted using Bannantine-Socie method can be seen in table 4 .

Table 4. Result comparison - Bannantine-Socie.

\begin{tabular}{|c|c|c|c|c|}
\hline \multicolumn{5}{|c|}{ Bannantine-Socie } \\
\hline Spec. nr. & $\begin{array}{c}\text { Load. } \\
\text { Hist. }\end{array}$ & Exp. & Findley & McDia. \\
\hline 16 & LH1 & 6520 & 15647 & 9146 \\
\hline C2 & LH1 & 6715 & 18152 & 10664 \\
\hline C4 & LH2 & 14480 & 21003 & 13084 \\
\hline
\end{tabular}

Fatigue life time estimation as a function of plane orientation for specimen n.16 (loaded with loading history 1) can be seen on Fig. 11 .

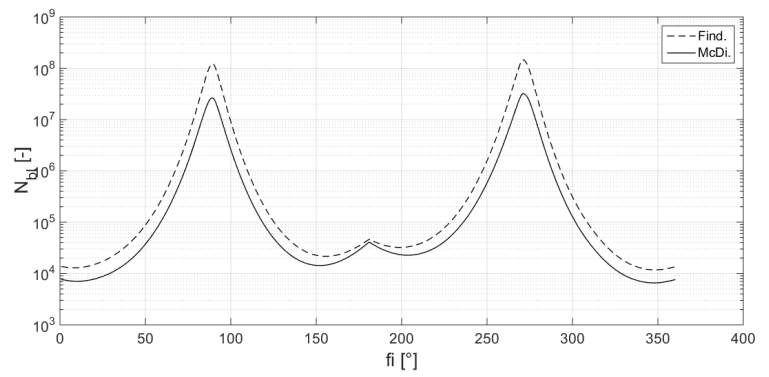

Fig.11. Fatigue life time as a function of plane orientation Bannantine-Socie method loading history LH1.

Fatigue life time estimation as a function of plane orientation for specimen n.C4 (loaded with loading history 2) can be seen on Fig. 12.

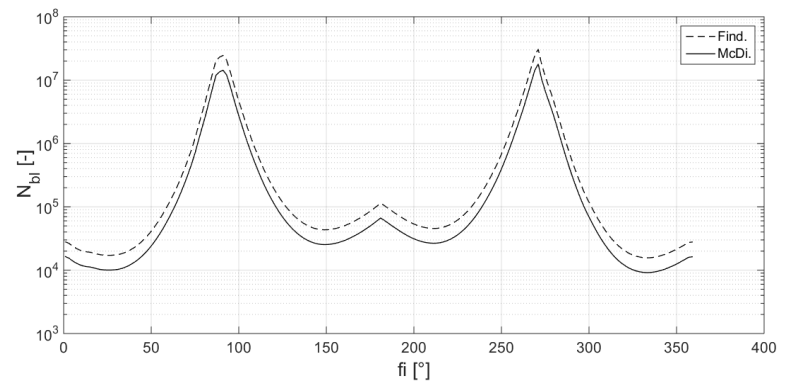

Fig.12. Fatigue life time as a function of plane orientation Bannantine-Socie method loading history LH2.

\section{Conclusion}

The fatigue life time estimation of testing specimens loaded with multiaxial non-proportional variable amplitude loading have was carried out in this paper. Two method of loading cycle identification and two different multiaxial criteria was used in the estimating process. The experimental verification of calculated results was realized.

The comparison between measurement and calculated prediction can be found in tables 3 and 4. An accuracy in percentage for all combination of chosen method can be found in table 5 .

Table 5. Performance of chosen method

\begin{tabular}{|c|c|c|c|}
\hline spec. $\mathbf{n .}$ & $\begin{array}{c}\text { Findley } \\
{[\%]}\end{array}$ & $\begin{array}{c}\text { McDiarmid } \\
{[\%]}\end{array}$ & method \\
\hline 16 & 180.7 & 100.8 & WB \\
\hline $\mathrm{C} 2$ & 240.5 & 132.6 & WB \\
\hline $\mathrm{C} 4$ & 117.8 & 66.2 & WB \\
\hline 16 & 240.0 & 140.3 & BS \\
\hline $\mathrm{C} 2$ & 270.3 & 158.8 & BS \\
\hline $\mathrm{C} 4$ & 145.0 & 90.4 & $\mathrm{BS}$ \\
\hline
\end{tabular}

Looking at the table 5, following conclusion can be stated:

- Both cycle counting method has similar percentage accuracy for chosen multiaxial damage models.

- The overall results show that Findley criterion underestimates loading conditions for both cycle counting method.

- McDiarmid criterion leads to the more accurate results for both cycle counting methods for the given loading condition.

- When using McDiarmid criterion Wang-Brown cycle counting method seems to be more accurate than Bannantine-Socie method for loading with zero mean stresses while BS method seems to be more accurate in case of non-proportional loadings with variable mean stresses.

- There should be noted the results from both counting method in combination with Mcdiarmid criterion fall within boundaries caused by scatter of material parameters. 
This work was supported by the Research \& Development Operational Programme funded by the ERDF ITMS: 26240220084 Science city Bratislava

\section{References}

1. J.A. Bannantine, D.F. Socie, A variable amplitude multiaxial fatigue life prediction method, in: Fatigue under biaxial and multiaxial loading, European Structural Integrity Society ESIS Publication 10, Mechanical engineering publications, London, 1991, pp. 367-370

2. C.H. Wang, M.W. Brown, Life prediction techniques for variable amplitude multiaxial fatigue - Part 1: Theories, J Eng Meter Technol 14 (1996) 367-370

3. C.H. Wang, M.W. Brown, Life prediction techniques for variable amplitude multiaxial fatigue - Part 2: Comparison with experimental results, J Eng Meter Technol 118 (1996) 371-374

4. W.N. Findley, Fatigue of metals under combinations of stresses, Trans ASME 79 (1957) 1337-1338

5. D.L. McDiarmid, A general criterion for high cycle multiaxial fatigue failure, Fatigue Fract Eng M 14, 4 (1991) 429-453

6. M. Margetin, R. Durka, V. Chmelko, Multiaxial fatigue criterion based on parameters from torsion and axial S-N curve, Fattura ed Integrita Strutturale 37, 10 (2016) 146-152

7. C.H. Wang, K.J. Miller, The effect of mean shear stress on torsion fatigue behavior, Fract Engng Mater Struct 14 (1991) 293-307 